\title{
Presencia de SARS-COV-2 en semen de hombres con COVID-19: revisión sistemática
}

\author{
G. Zulema Zeñas*1,a
}

RESUMEN

La pandemia de la COVID-19 ha causado un impacto aún desconocido en la salud reproductiva masculina. Por ello, el objetivo de esta revisión fue recolectar evidencia para descubrir si el agente causante de la COVID-19 puede encontrarse en el semen. La búsqueda se realizó en las bases de datos PubMed, BVS y Google Scholar, e incluyó los artículos que mostraban los resultados del análisis de semen realizado para encontrar el virus SARS-CoV-2 en hombres diagnosticados con COVID-19. Once estudios fueron seleccionados para esta revisión que aportaron 296 muestras. De ellas, solo el $2,70 \%$ fueron positivas. Además, se encontraron resultados mixtos respecto a la morfología de los espermatozoides, y también pacientes con azoospermia u oligospermia. Este último hallazgo podría ser consecuencia del elevado número de receptores de la enzima convertidora de angiotensina 2 (ECA 2) que existen en los testículos, lo que hace a estos órganos un blanco del virus SARS-CoV-2. Se concluye que debido al limitado número de estudios y muestras aún no es posible responder la pregunta de estudio planteada.

Palabras clave: Semen; Infecciones por Coronavirus, Análisis de Semen (Fuente: DeCS BIREME).

\section{Presence of SARS-CoV-2 in semen of men with COVID-19: a systematic review}

\section{ABSTRACT}

The COVID-19 pandemic has caused an unknown impact on the male reproductive health. Therefore, this article aimed to collect evidence to discover if the causative agent of COVID-19 is found in semen. A search was carried out in the PubMed, BVS and Google Scholar databases. Articles with results of semen analyses to determine the presence of SARS-CoV-2 in men diagnosed with COVID-19 were considered. Eleven studies with a total of 296 samples were collected in this review, out of which only $2.70 \%$ showed positive results. In addition, mixed results regarding the morphology of the spermatozoa, as well as patients with azoospermia or oligospermia, were reported. The latter could be a consequence of the high levels of the angiotensin converting enzyme 2 (ACE2) in the testes, making them a target organ for SARS-CoV-2. It is concluded that, due to the limited number of studies and samples, it is still not possible to answer this study question.

Keywords: Semen; Coronavirus infections; Semen analysis (Source: MeSH NLM).

1 Universidad Ricardo Palma, Facultad de Medicina Humana. Lima, Perú.

a Estudiante de Medicina.

*Autor corresponsal. 


\section{INTRODUCCIÓN}

La pandemia del COVID-19 causada por el virus SARS-CoV- 2 ha tenido repercusión en todo el mundo, por lo que se trata de mitigarla con gran ahínco ${ }^{(1)}$. A pesar de todos los esfuerzos, este virus ha causado un impacto aún inmensurable en la salud reproductiva masculina. Aunque el SARS-CoV-2 se transmite sobre todo mediante el contacto directo y a través de gotículas respiratorias que poseen un diámetro de 5 a 10 micrómetros ${ }^{(2-4)}$, el virus se ha detectado también en muestras de sangre, esputo, orina, líquido de lavado broncoalveolar e hisopos anales y nasales ${ }^{(5,6)}$, lo que podría sugerir que existen otras vías de infección. El SARS-CoV-2 es similar en estructura del genoma, transmisión y patogenia, al SARS-CoV ${ }^{(7)}$, el cual, según el estudio de Xu et al. ${ }^{(8)}$, causó orquitis y afectó significativamente los testículos en un grupo de pacientes.

Debido a que los hombres son el grupo en que el virus muestra una mayor incidencia y letalidad ${ }^{(9,10)}$, probablemente debido a factores como la alta expresión de los receptores de coronavirus, la enzima convertidora de angiotensina 2 (ECA 2) y las hormonas sexuales ${ }^{(11)}$, surge la interrogante de si es posible encontrar el SARS-CoV-2 en el semen, como en el caso de otros virus como zika, paperas, citomegalovirus y ébola ${ }^{(12-14)}$, los cuales pueden atravesar la barrera hemato-testicular y provocar una respuesta inmunitaria dentro del testículo ${ }^{(15)}$. Por ello el objetivo de este artículo fue recopilar los resultados de estudios acerca de la presencia de SARS-CoV-2 en el semen humano.

\section{ESTRATEGIA DE BÚSQUEDA}

La revisión fue efectuada utilizando la metodología de revisión sistemática ${ }^{(16)}$. Los artículos relacionados con el COVID-19 y el semen humano se buscaron en las bases de datos BVS, PubMed y Google Scholar. En las dos primeras, la pesquisa electrónica se realizó mediante distintos términos de búsqueda; y en Google Scholar, se empleó una combinación de palabras clave relacionadas.

Los criterios de inclusión aplicados fueron los siguientes: artículos en idiomas inglés o español que incluyeran el texto completo y que contengan resultados de análisis de semen humano en el que investigaron la presencia de SARS-CoV-2 en hombres diagnosticados con COVID-19. Se excluyeron las cartas al editor y los estudios realizados en animales.

\section{RESULTADOS}

Al tratarse de un tema novedoso fueron pocos los artículos encontrados acerca del SARS-CoV-2 y que además abordaran el análisis de semen humano o la salud reproductiva masculina.

En la figura 1 se muestra el diagrama de flujo según el modelo PRISMA ${ }^{(17)}$, que muestra el proceso de selección de artículos.

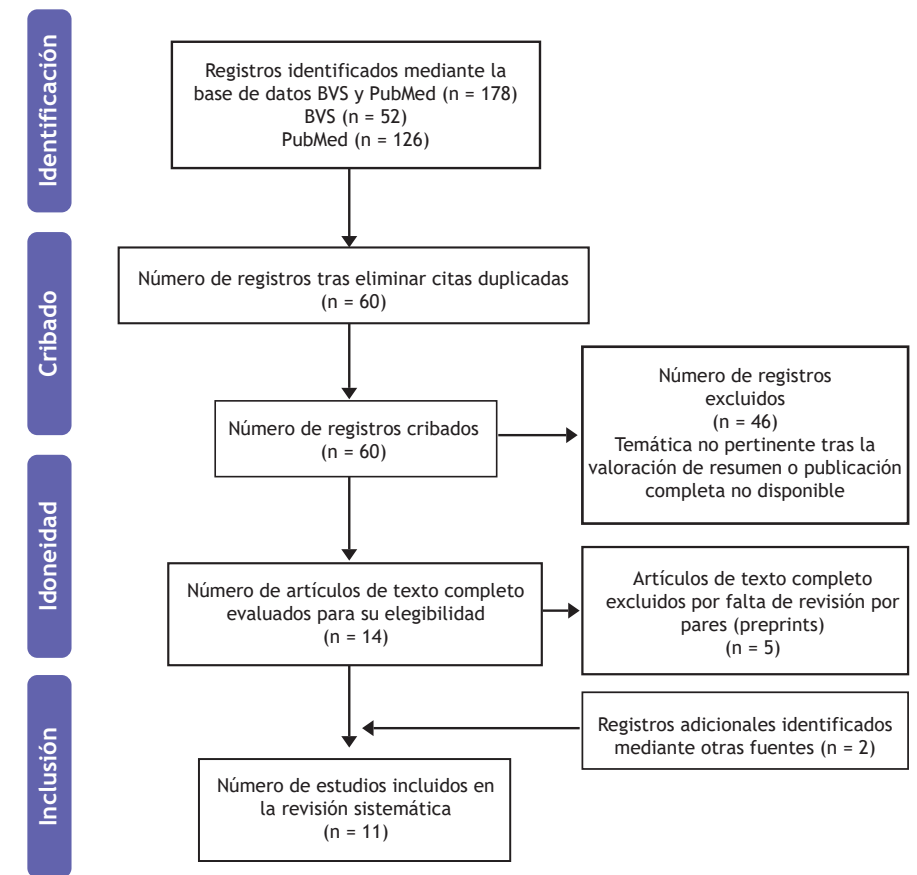

Figura 1. Diagrama de flujo que muestra el proceso de selección de artículos 
Al momento de la revisión, existían once estudios (18-28): ocho de cohorte, uno observacional, un reporte de caso y un estudio piloto (Tabla 1), que reportan la evaluación del semen de hombres infectados con SARS-CoV-2. En total, 296 muestras fueron recolectadas en conjunto, tomadas desde el día 1 hasta 112 días después de que el paciente mostró un resultado positivo a una prueba RT-PCR nasofaríngea para SARS-CoV-2 (Figura 2), y todas, excepto ocho, fueron negativas para la presencia del virus ${ }^{(2)}$.

Tabla 1. Características de los estudios incluidos en la revisión

\begin{tabular}{|c|c|c|c|c|c|c|c|}
\hline \multirow[t]{2}{*}{ Autor } & \multirow[t]{2}{*}{$\begin{array}{l}\text { Tipo de } \\
\text { estudio }\end{array}$} & \multirow[t]{2}{*}{ País } & \multirow{2}{*}{$\begin{array}{l}\mathrm{N} .^{\circ} \text { de } \\
\text { muestras } \\
\text { recolectadas }\end{array}$} & \multirow{2}{*}{$\begin{array}{c}\text { Tiempo de } \\
\text { recolección de } \\
\text { semen (en días) }\end{array}$} & \multirow{2}{*}{$\begin{array}{l}\text { Media } \\
\text { de edad } \\
\text { (Min-Máx) }\end{array}$} & \multicolumn{2}{|c|}{$\begin{array}{l}\text { Resultado } \\
\text { de RT-PCR }\end{array}$} \\
\hline & & & & & & Positivo & Negativo \\
\hline Kayaaslan et al. & Cohorte & Turquía & 16 & $0-7$ & $33,5(18-64)$ & 16 & 0 \\
\hline Pan et al. & Observacional & China & 34 & $29-36$ & $37(18-57)$ & 34 & 0 \\
\hline Holtmann et al. & Cohorte & Alemania & 20 & $8-54$ & 42,2 & 20 & 0 \\
\hline Li et al. & Cohorte & China & 38 & NE & NE & 32 & 6 \\
\hline Paoli et al. & Reporte de caso & Italia & 1 & 8 & 38 & 1 & 0 \\
\hline Temiz et al. & Piloto & Turquía & 20 & 5 & $37,21 \pm 8,59$ & 20 & 0 \\
\hline Guo et al. & Cohorte & China & 21 & 32 & $41(20-62)$ & 21 & 0 \\
\hline Burke et al. & Cohorte & Estados Unidos & 18 & $1-28$ & $32(24-57)$ & 18 & 0 \\
\hline Machado et al. & Cohorte & Estados Unidos & 15 & 14 & $23,26(19-43)$ & 14 & 1 \\
\hline Gacci et al. & Cohorte & Italia & 43 & $1-112$ & $18-65$ & 40 & 1 \\
\hline Ruan et al. & Cohorte & China & 70 & $3-65$ & $31(21-49)$ & 70 & 0 \\
\hline
\end{tabular}

NE: no especificado

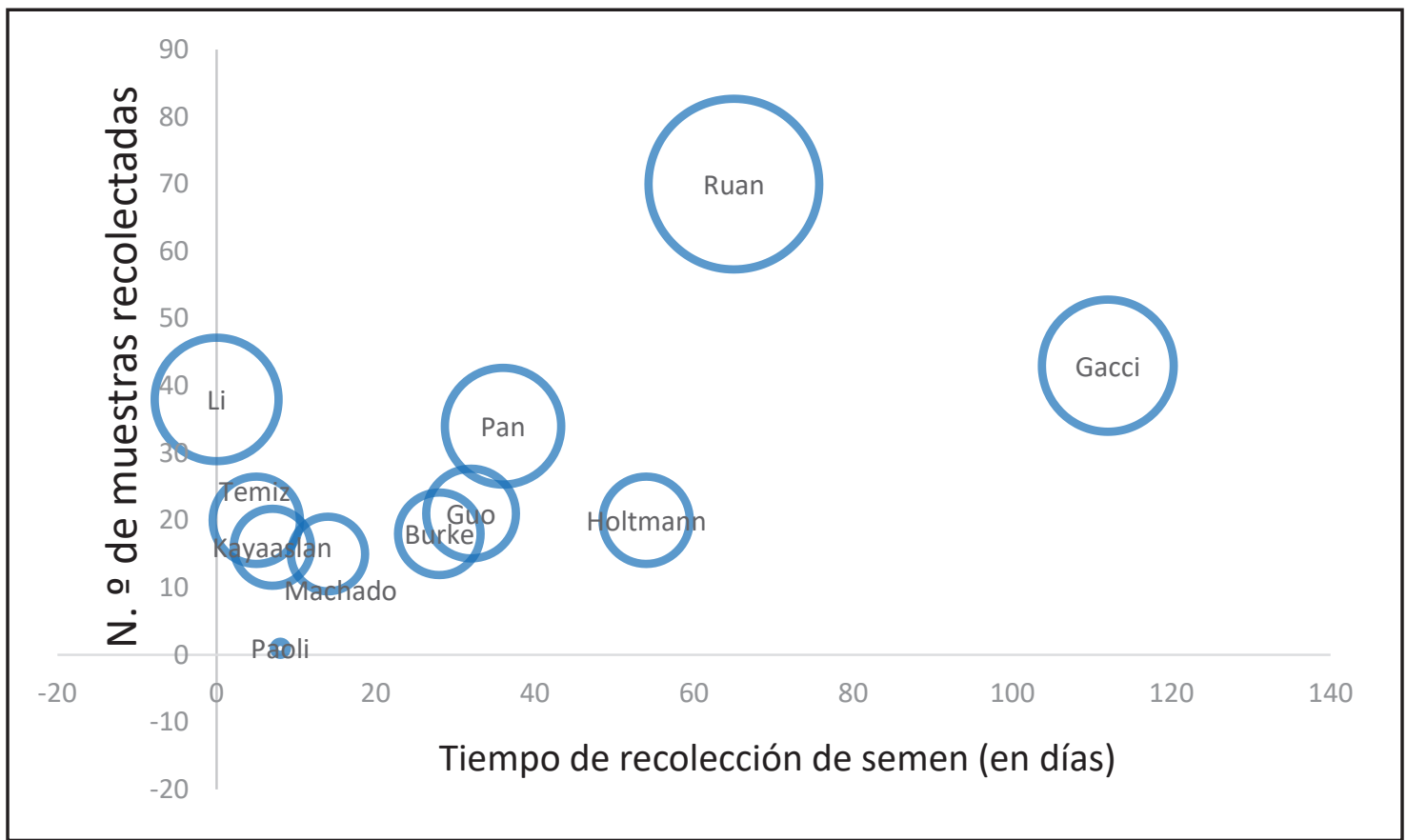

Figura 2. Diagrama de dispersión del número de muestras recolectadas y el tiempo de recolección de semen. El tamaño de cada punto de datos indica el tamaño de muestra correspondiente 
El 36,36\% de los estudios ( $n=4$ ) se realizaron en China; el $18,18 \%(n=2)$, en Estados Unidos; otro 18,18 \% ( $n=2)$, en Turquía; $9,09 \%(n=1)$, en Alemania; y finalmente $18,18 \%$ $(n=2)$, en Italia.

En la investigación de Kayaaslan B et al. (2020) (18) se analizó el semen de 16 pacientes de 33,5 años, en promedio, hospitalizados con infección confirmada por SARS-COV-2, que carecían de antecedentes o síntomas de enfermedad urogenital, así como de síntomas respiratorios (a excepción de uno). Todas las muestras de semen fueron tomadas mientras los pacientes se encontraban en la etapa aguda de la infección y resultaron negativas para la PCR del SARS-CoV-2.

Pan et al. (2020) (19) realizaron una prueba con qRT-PCR, en búsqueda del virus SARS-CoV-2, al semen de 34 varones de entre 18 y 57 años. Las pruebas se llevaron a cabo de 29 a 36 días después dar positivo al virus con un análisis de frotis faríngeo. Todos los resultados fueron negativos.

En una serie de casos, Holtmann et al. (2020) describieron el análisis del semen de 34 varones con una edad promedio de 42,2 años, que fueron calificados como pacientes en convalecencia (pacientes infectados por SARS-CoV-2 confirmado mediante la prueba RT-PCR o anticuerpos), grupo control negativo (conformado por 14 varones) y pacientes con infección aguda. En este estudio, el tiempo promedio de obtención de una muestra de semen tras la finalización de los síntomas fue de 32,7 días, y no se detectó SARS-CoV-2 mediante RT-PCR en el semen de hombres convalecientes y con infección aguda.

Li et al. (2020) (21) informaron acerca de 38 participantes que proporcionaron una muestra de semen para ser analizada. Del total de participantes, 23 habían logrado la recuperación clínica y 15 se encontraban en la etapa aguda de la infección. Los resultados de las pruebas de semen mostraron que seis pacientes obtuvieron resultados positivos para el SARS-CoV-2, cuatro de ellos se hallaban en la etapa aguda de la infección y dos estaban recuperándose. Paoli et al. (2020) (22) informaron el caso de un varón de 31 años con fiebre, anosmia, mialgia y ageusia que fue evaluado y tuvo un resultado y positivo para SARS-CoV-2 a través de un frotis faríngeo. El hombre proporcionó muestras de semen y orina ocho días después, en las que se midió la presencia de ARN viral mediante un sistema de RT-PCR, con un resultado negativo.

De igual manera, la investigación de Temiz et al. (2020) (23) evaluó las muestras de semen de 30 hombres catalogados en 3 grupos (una decena por cada grupo): pacientes con COVID-19 antes del tratamiento, pacientes confirmados de COVID-19 después del tratamiento y un grupo de controles. Los resultados describieron una disminución significativa en el porcentaje de morfología normal, así como la ausencia de ARN de SARS-CoV-2 en el análisis de semen de los pacientes del primer y segundo grupos.

El estudio de Guo et al. (2020) (24) incluyó 23 hombres con resultados positivos para el SARS-CoV-2, mediante amplificación qRT-PCR en muestras de frotis faríngeo, pero solo se obtuvieron 21 muestras de semen: en ninguna de ellas encontraron SARS-CoV-2 después de una mediana de 32 días, desde la confirmación del diagnóstico, lo que interpretaron como una baja posibilidad de transmisión sexual a través del semen, alrededor de un mes después de la primera detección. Cabe resaltar que, en todas las muestras de semen analizadas en este estudio, el recuento total de espermatozoides, el recuento total de espermatozoides móviles y la distribución de la morfología de los espermatozoides estaban dentro de los rangos normales.

Recientemente, Burke et al. (2021) (25) informaron los resultados negativos del análisis de semen de 18 pacientes que se sometieron a extracción de ARN viral y procesados mediante RT-PCR para detectar el SARS-CoV-2. Concluyeron que el virus se encuentra ausente en el semen durante la fase aguda o la convalecencia de la COVID-19.

Por otro lado, Machado et al. (2021) (26) incluyeron a 15 pacientes diagnosticados con un hisopado nasal positivo para SARS-CoV-2. Luego, recuperaron una muestra de semen después de una ducha (con la finalidad de evitar la contaminación cruzada) y se analizó el ARN viral en el semen. Las muestras se analizaron con RT-PCR para detectar el ARN viral y se volvieron a analizar 24 horas después de la primera prueba. El ARN viral del SARS-CoV-2 estuvo presente en un paciente. Los investigadores concluyeron que es posible aislar el virus del semen humano y alertaron sobre una posible nueva forma de transmisión de la enfermedad.

La investigación de Ruan et al. (2020) ${ }^{(28)}$ describió el análisis del semen de 74 pacientes con COVID-19 ya recuperados, con una mediana de edad de 31 años. El intervalo medio entre la recolección de muestras de semen y la última prueba de RT-PCR con hisopo faríngeo positivo fue de 80 días y no se detectó SARS-CoV-2 mediante RT-PCR en ninguna de las 70 muestras. Los resultados describen que la concentración de esperma mostraba una tendencia decreciente con la severidad de la enfermedad, así como el hallazgo de que los pacientes con un tiempo de recuperación mayor a 90 días tuvieron un recuento total de espermatozoides significativamente menor, en comparación con los pacientes con un tiempo de recuperación más corto.

Finalmente, Gacci et al. (2021) ${ }^{(27)}$ reportaron los resultados de 43 pacientes varones infectados por COVID-19. Doce de ellos no fueron hospitalizados, 26 ingresaron a la Unidad de Medicina Interna y 5 ingresaron a la Unidad de Cuidados Intensivos (UCI). El ARN del SARS-COV-2 fue encontrado en la 
muestra de un paciente, quien había sido ingresado a la $\mathrm{UCl}$ durante la infección por COVID-19 y cuya pareja (a pesar de haber tenido sexo oral, vaginal y anal sin protección con el infectado) tuvo resultados negativos en hisopados faríngeos, vaginales y rectales. El análisis de semen demostró que ocho varones eran azoospérmicos y tres, oligospérmicos. Además, los resultados revelaron que el estado de azoospermia estaba altamente relacionado con la severidad de la enfermedad. El análisis también detectó la presencia de leucocitos en el semen del $37,2 \%$ de los pacientes, y que el $76,7 \%$ mostraban niveles patológicos de IL-8.

\section{DISCUSIÓN}

En este artículo se ha revisado la presencia de SARS-CoV-2 en el semen de hombres diagnosticados con COVID-19. De los once estudios seleccionados, solo el $27,27 \%$ de ellos reportaron, al menos, un resultado positivo ${ }^{(21,26,28)}$, y del total de muestras recolectadas en combinación, tan solo en $8(2,70 \%)$ se detectó la presencia del ARN viral con la prueba RT-PCR, lo que sugiere una posibilidad extremadamente baja de encontrar SARS-CoV-2 en el semen.

Por otro lado, también es importante resaltar los resultados mixtos de la morfología de los espermatozoides. Mientras que el estudio de Guo et al. (21) mostró que la morfología y el recuento total de espermatozoides de hombres diagnosticados con COVID-19 estaban dentro de los rangos normales, otros trabajos revelaban anormalidades en el recuento total, morfología y concentración de espermatozoides (23,24,27,28). Referente a ello, Ruan et al. (27) describieron una relación de estos cambios en la concentración de esperma y recuento total de espermatozoides con la severidad y el tiempo de recuperación del paciente. De igual forma, Gacci et al. reportaron pacientes azoospérmicos y oligoespérmicos $y$, además, informaron que la condición de azoospermia estaba altamente relacionada con la severidad de la enfermedad ${ }^{(28)}$.

Esta revisión muestra que existe una mínima presencia de SARS-CoV-2 en el semen, al igual que Gonzalez et al. ${ }^{(29)}$, quienes han tratado de dilucidar si es posible o no encontrar el ARN del virus en el semen de hombres infectados. Aún son pocas las muestras estudiadas para considerar que estos datos son concluyentes y la cantidad de investigaciones que incluyeron participantes en la etapa aguda de infección es baja $(18,20,21,25)$. Todo ello representa una limitación importante, ya que se considera la etapa aguda de la enfermedad como el periodo de mayor transmisibilidad viral y, por ende, el momento preciso para detectar el virus. Por otro lado, Gacci et al. (27) muestran evidencia que podría esclarecer la pregunta de si es posible la trasmisión sexual del COVID-19: en sus resultados, un hombre cuya muestra de semen fue positiva no infectó a su pareja a pesar de haber mantenido relaciones sexuales sin protección.

Ante estos hallazgos, surge la pregunta acerca de cuáles son los tejidos y células del aparato reproductor masculino involucrados. Los testículos (células de Leydig, los espermatozoides o las células de Sertoli), glándulas accesorias y el tracto urinario son los principales candidatos ${ }^{(29)}$. Se sabe que los receptores ECA2 facilitan la entrada y replicación celular del SARS-CoV-2, lo que sugiere que las células con gran número de estos receptores son las más vulnerables a la infección por COVID-19 ${ }^{(30-32)}$. En los testículos, las células de Sertoli tienen el nivel de expresión de ECA2 más alto ${ }^{(33-34)}$. Respecto al número de espermatozoides de los hombres infectados por COVID-19, la investigación de Moghimi et al. demostró que la infección del virus SARS-CoV-2 provoca una interrupción de la espermatogénesis mediante la vía del estrés oxidativo y luego induce la apoptosis. Shen at al. observaron altos niveles de expresión del receptor ECA2 en testículos de pacientes infértiles en comparación con hombres normales, lo que sugiere que el virus SARS-CoV- 2 puede causar trastornos reproductivos a través de la activación anormal de la vía ECA2 ${ }^{(35,36)}$.

Para evaluar la presencia de SARS-CoV-2 en el semen y descartar a la COVID-19 como una enfermedad de transmisión sexual, es necesario investigar a los pacientes durante la fase aguda de la infección y hacer un seguimiento a largo plazo de los hombres que tuvieron de un tiempo de recuperación prolongado. Así mismo, es indispensable seguir investigando para determinar una potencial asociación del deterioro de la calidad del esperma e infertilidad con la infección con el SARS-CoV-2.

\section{CONCLUSIONES}

Diversas investigaciones reportan la ausencia de SARS-CoV-2 en el semen humano y otros pocos, su presencia. Aunque existe evidencia que podría descartar al COVID-19 como una enfermedad de transmisión sexual, aún hacen falta investigaciones. Finalmente, algunos estudios revelan un efecto negativo de la COVID-19 en la salud sexual masculina, cuyos alcances es necesario investigar, ya que entender el mecanismo del virus ayudará a determinar posibles nuevas medidas preventivas.

Contribuciones de los autores: La autora participó de la génesis de la idea, diseño del proyecto, desarrollo, recolección e interpretación de datos, análisis de los resultados y preparación del manuscrito.

Fuentes de financiamiento: El artículo ha sido financiado por la autora.

Conflicto de intereses: La autora declara no tener ningún conflicto de intereses. 


\section{REFERENCIAS BIBLIOGRÁFICAS}

1. World Health Organization. Novel Coronavirus (2019-nCoV): situation report, 1 [Internet]. WHO; 2020. Disponible en: https://apps.who. int/iris/handle/10665/330760

2. Li Q, Guan X, Wu P, Wang X, Zhou L, Tong Y, et al. Early transmission dynamics in Wuhan, China, of novel Coronavirus-infected pneumonia. N Engl J Med. 2020; 382(13): 1199-207.

3. Burke RM, Midgley CM, Dratch A, Fenstersheib M, Haupt T, Holshue $M$, et al. Active monitoring of persons exposed to patients with confirmed COVID-19 - United States, January-February 2020. MMWR Morb Mortal Wkly Rep. 2020; 69(9): 245-6.

4. Organización Mundial de la Salud. Vías de transmisión del virus de la COVID-19: repercusiones para las recomendaciones relativas a las precauciones en materia de prevención y control de las infecciones [Internet]. OMS; 2020. Disponible en: https://www.who.int/es/ news-room/commentaries/detail/modes-of-transmission-of-viruscausing-covid-19-implications-for-ipc-precaution-recommendations

5. Peng L, Liu J, Xu W, Luo Q, Chen D, Lei Z, et al. SARS-CoV-2 can be detected in urine, blood, anal swabs, and oropharyngeal swabs specimens. J Med Virol. 2020; 92(9): 1676-80.

6. Wang W, Xu Y, Gao R, Lu R, Han K, Wu G, et al. Detection of SARS CoV-2 in different types of clinical specimens. JAMA. 2020; 323(18): 1843-4.

7. Yan Y, Chang L, Wang L. Laboratory testing of SARS-CoV, MERSCoV, and SARS-CoV-2 (2019-nCoV): Current status, challenges, and countermeasures. Rev Med Virol. 2020; 30(3): e2106.

8. Xu J, Qi L, Chi X, Yang J, Wei X, Gong E, et al. Orchitis: a complication of severe acute respiratory syndrome (SARS). Biol Reprod. 2006; 74(2): 410-6.

9. Guan WJ, Ni ZY, Hu Y, Liang WH, Ou CQ, He JX, et al. Clinical characteristics of Coronavirus Disease 2019 in China. N Engl J Med. 2020; 382(18): 1708-20.

10. Mangia C, Russo A, Civitelli S, Gianicolo EAL. Sex/gender differences in COVID-19 lethality: what the data say, and do not say. Epidemiol Prev. 2020; 44(5-6 Suppl 2): 400-6.

11. Bwire GM. Coronavirus: Why men are more vulnerable to Covid-19 than women?. SN Compr Clin Med. 2020; 1-3.

12. Salam AP, Horby PW. The breadth of viruses in human semen. Emerg Infect Dis. 2017; 23(11): 1922-4.

13. Mansuy JM, Suberbielle E, Chapuy-Regaud S, Mengelle C, Bujan L, Marchou B, et al. Zika virus in semen and spermatozoa. Lancet Infect Dis. 2016; 16(10): 1106-7.

14. Brainard J, Pond K, Hooper L, Edmunds K, Hunter P. Presence and persistence of Ebola or Marburg virus in patients and survivors: A rapid systematic review. PLoS Negl Trop Dis. 2016; 10(2): e0004475.

15. Zhao S, Zhu W, Xue S, Han D. Testicular defense systems: immune privilege and innate immunity. Cell Mol Immunol. 2014; 11(5): 428-37.

16. Munn Z, Peters MDJ, Stern C, Tufanaru C, McArthur A, Aromataris E. Systematic review or scoping review? Guidance for authors when choosing between a systematic or scoping review approach. BMC Med Res Methodol. 2018; 18(1): 143.

17. Moher D, Liberati A, Tetzlaff J, Altman DG, The PRISMA Group. Preferred reporting items for systematic reviews and meta-analyses: the PRISMA statement. PLoS Med. 2009; 6(7): e1000097.

18. Kayaaslan B, Korukluoglu G, Hasanoglu I, Kalem AK, Eser F, Akinci E, et al. Investigation of SARS-CoV-2 in semen of patients in the acute stage of COVID-19 infection. Urol Int. 2020; 104(9-10): 678-83.

19. Pan F, Xiao X, Guo J, Song Y, Li H, Patel DP, et al. No evidence of severe acute respiratory syndrome-coronavirus 2 in semen of males recovering from coronavirus disease 2019. Fertil Steril. 2020; 113(6): 1135-9.

20. Holtmann N, Edimiris P, Andree M, Doehmen C, Baston-Buest D,
Adams 0, et al. Assessment of SARS-CoV-2 in human semen-a cohort study. Fertil Steril. 2020; 114(2): 233-8.

21. Li D, Jin M, Bao P, Zhao W, Zhang S. Clinical characteristics and results of semen tests among men with Coronavirus disease 2019. JAMA Netw Open. 2020; 3(5): e208292.

22. Paoli D, Pallotti F, Colangelo S, Basilico F, Mazzuti L, Turriziani O, et al. Study of SARS-CoV-2 in semen and urine samples of a volunteer with positive naso-pharyngeal swab. J Endocrinol Invest. 2020; 43(12): 1819-22.

23. Temiz MZ, Dincer MM, Hacibey I, Yazar RO, Celik C, Kucuk SH, et al. Investigation of SARS-CoV-2 in semen samples and the effects of COVID-19 on male sexual health by using semen analysis and serum male hormone profile: A cross-sectional, pilot study. Andrologia. 2021; 53(2): e13912.

24. Guo L, Zhao S, Li W, Wang Y, Li L, Jiang S, et al. Absence of SARSCoV-2 in semen of a COVID-19 patient cohort. Andrology. 2021; 9(1): $42-7$.

25. Burke CA, Skytte AB, Kasiri S, Howell D, Patel ZP, Trolice MP, et al. A cohort study of men infected with COVID-19 for presence of SARSCoV-2 virus in their semen. J Assist Reprod Genet. 2021; 38(4): 785-9.

26. Machado B, Barra GB, Scherzer N, Massey J, Luz HDS, Jacomo RH, et al. Presence of SARS-CoV-2 RNA in semen-cohort study in the United States COVID-19 positive patients. Infect Dis Rep. 2021; 13(1): 96-101.

27. Gacci M, Coppi M, Baldi E, Sebastianelli A, Zaccaro C, Morselli S, et al. Semen impairment and occurrence of SARS-CoV-2 virus in semen after recovery from COVID-19. Hum Reprod. 2021; 36(6): 1520-9.

28. Ruan Y, Hu B, Liu Z, Liu K, Jiang H, Li H, et al. No detection of SARS-CoV-2 from urine, expressed prostatic secretions, and semen in 74 recovered COVID-19 male patients: A perspective and urogenital evaluation. Andrology. 2021; 9(1): 99-106.

29. Gonzalez DC, Khodamoradi K, Pai R, Guarch K, Connelly ZM, Ibrahim $E$, et al. A systematic review on the investigation of SARS-CoV-2 in semen. Res Rep Urol. 2020; 12: 615-21.

30. Massarotti C, Garolla A, Maccarini E, Scaruffi P, Stigliani S, Anserini $P$, et al. SARS-CoV-2 in the semen: Where does it come from?. Andrology. 2021; 9(1): 39-41.

31. Lukassen S, Chua RL, Trefzer T, Kahn NC, Schneider MA, Muley T, et al. SARS-CoV-2 receptor ACE2 and TMPRSS2 are primarily expressed in bronchial transient secretory cells. EMBO J. 2020; 39(10): e105114.

32. Cheng H, Wang Y, Wang GQ. Organ-protective effect of angiotensinconverting enzyme 2 and its effect on the prognosis of COVID-19. J Med Virol. 2020; 92(7): 726-30.

33. Fu J, Zhou B, Zhang L, Balaji KS, Wei C, Liu X, et al. Expressions and significances of the angiotensin-converting enzyme 2 gene, the receptor of SARS-CoV-2 for COVID-19. Mol Biol Rep. 2020; 1-10.

34. Liu X, Chen Y, Tang W, Zhang L, Chen W, Yan Z, et al. Single-cell transcriptome analysis of the novel coronavirus (SARS-CoV-2) associated gene ACE2 expression in normal and non-obstructive azoospermia (NOA) human male testes. Sci China Life Sci. 2020; 63(7): 1006-15.

35. Shen Q, Xiao X, Aierken A, Yue W, Wu X, Liao M, et al. The ACE2 expression in Sertoli cells and germ cells may cause male reproductive disorder after SARS-CoV-2 infection. J Cell Mol Med. 2020; 24(16): 9472-7.

36. Moghimi N, Farsani BE, Ghadipasha M, Mahmoudiasl GR, Piryaei A, Aliaghaei A, et al. COVID-19 disrupts spermatogenesis through the oxidative stress pathway following induction of apoptosis. Apoptosis. $2021 ; 1-16$. 
Correspondencia:

G. Zulema Zeñas

Dirección: Av. Oscar R.Benavides 3008, Block E Dpto 101

Etapa III, Cercado de Lima. Lima, Perú.

Teléfono: 979362373

Correo electrónico: 201411910@urp.edu.pe

Recibido: 31 de marzo de 2021

Evaluado: 23 de abril de 2021

Aprobado: 07 de julio de 2021

( $)$ La revista. Publicado por Universidad de San Martín de Porres, Perú. (cc) Br Licencia de Creative Commons Artículo en acceso abierto bajo términos de Licencia Creative Commons Atribución 4.0 Internacional. (http://creativecommons.org/licenses/by/4.0/)

ORCID iDs

G. Zulema Zeñas ( https:// orcid.org/0000-0002-3059-8184 